\title{
ON THE EXISTENCE OF RIGID COMPACT ORDERED SPACES
}

\author{
J. DE GROOT AND M. A. MAURICE
}

1. It is easily seen that every compact ordered space with infinitely many points which has a countable base admits continuously many autohomeomorphisms. For, if there are countably many isolated points, the assertion is obvious. In the other case the assertion follows from the fact that there is either a separable connected subspace which is consequently homeomorphic to an interval of the real numbers or the space is zero-dimensional and so is homeomorphic to the Cantor set (possibly except for finitely many isolated points). Jónsson [1] and Rieger [2] each give an example of an infinite zerodimensional compact ordered space $S$, which is rigid, i.e. a space such that the only homeomorphism of $S$ onto $S$ is the identity mapping. However, the weight of the constructed space $S$ is very large. (Here weight means the minimal cardinality of an open base.) The purpose of this note is to show the existence of (a family of $2^{c}$ ) rigid zerodimensional compact ordered spaces each having continuous weight and continuous power. (Here $c$ denotes the cardinal of the set of real numbers.)

2. If $N$ is a set and $f$ is a map of a subset $S$ of $N$ into $N$, then for every $M \subset N$ we write $f M$ instead of $f[S \cap M]$.

In de Groot [1] the following concepts were introduced:

(a). If $f$ is a map of $S \subset N$ into $N$, then $f$ is called a displacement of order $m$, if there exists a subset $V$ of $N$ such that

$$
V \cap f V=\varnothing, \quad|f V|=m,
$$

whereas for no $n>m$ there is a subset $W$ of $N$, such that

$$
W \cap f W=\varnothing, \quad|f W|=n .
$$

(b). If $N$ is a topologicals pace, then $f$ is called a continuous displacement of $S \subset N$, if $f$ is a continuous map of $S$ into $N$ and $f$ is a displacement of order $c$.

One easily proves the following generalization of [1, Lemma 2].

Lemma. Let $P$ be a separable metric space. Let $Q$ be a subset of $P$ of which every point is a point of condensation in $Q$.

Presented to the Society, January 27, 1965 under the title $A$ rigid zero-dimensional compact ordered space of continuous power and continuous weight; received by the editors October 26, 1965. 
If $\psi: Q \rightarrow P$ is a map ( $\neq$ identity) such that every point of $\psi[Q]$ is again a point of condensation in $\psi[Q]$, then $\psi$ is a continuous displacement in $P$.

Strengthening in a trivial way the result of [1, Theorem 1], for the case that $M=$ the unit interval $I$ of the reals and $\left\{K_{\beta}\right\}$ is the system of all uncountable compact subsets of $I$ (cf. [1, Theorem 2]), we obtain the following theorem.

Theorem A. There exists a family $\left\{F_{\gamma}\right\}$ of $2^{c}$ zero-dimensional subsets of $I$, each of power $c$, such that

(i) every subinterval of $I$ contains $c$ points of each $F_{\gamma}$ and of each $I \backslash F_{\gamma}$ (in particular $\bar{F}_{\gamma}=I$ for all $\gamma$ ).

(ii) $\left|F_{\gamma} \backslash F_{\gamma^{\prime}}\right|=c$ for all $\gamma, \gamma^{\prime}$ with $\gamma \neq \gamma^{\prime}$.

(iii) $\left|\phi F_{\gamma} \backslash F_{\gamma^{\prime}}\right|=c$ for all $\gamma, \gamma^{\prime}$ and all continuous displacements $\phi$ of $F_{\gamma}$ in $I$.

From this theorem it follows [1, Theorem 2], that there exists neither a proper autohomeomorphism of any $F_{\gamma}$, nor a homeomorphism of any $F_{\gamma}$ onto some other $F_{\gamma^{\prime}}$. (For, such a map would be a continuous displacement of $F_{\gamma}$ (cf. [1, Lemma 2]), which is impossible according to Theorem A(iii).)

3. Define the set

$$
S_{\gamma}=F_{\gamma} \cup\{(b, 0),(b, 1)\}_{b \in I \backslash \boldsymbol{F} \gamma}
$$

and introduce an order in it in the natural way (i.e. if $\tilde{p}=p$ when $p \in F_{\gamma}$ and $\tilde{p}=b$ when $p=(b, 0)$ or $p=(b, 1)$, then $p<q$ in $S$ if $\tilde{p}<\tilde{q}$ in $I$; and moreover $(b, 0)<(b, 1)$ for every $\left.b \in I \backslash F_{\gamma}\right)$. It is clear that $S_{\gamma}$ (supplied with its interval topology) is a separable zero-dimensional compact space of weight $c$.

Every subspace of $S_{\gamma}$ which contains $c$ points of $S_{\gamma} \backslash F_{\gamma}$ has weight $c$. And $F_{\gamma}$-as-a-subspace-of- $I$ is homeomorphic to $F_{\gamma}$-as-a-subspace-of- $S_{\gamma}$.

THEOREM B. $S_{\gamma}$ is rigid for every $\gamma$.

$S_{\gamma}$ is not homeomorphic to $S_{\gamma^{\prime}}$, if $\gamma \neq \gamma^{\prime}$.

Proof. Let $\phi_{\gamma}$ be the continuous map of $S_{\gamma}$ onto $I$ which is defined by

$$
\begin{array}{cl}
\phi_{\gamma}(a)=a, & \text { if } a \in F_{\gamma}, \\
\phi_{\gamma}((b, 0))=\phi_{\gamma}((b, 1))=b, & \text { if } b \in I \backslash F_{\gamma} .
\end{array}
$$

Now suppose that $f_{\gamma}$ is a proper autohomeomorphism of $S_{\gamma}$, and that 
$f_{\gamma \gamma^{\prime}}$ is a homeomorphism of $S_{\gamma}$ onto $S_{\gamma^{\prime}}\left(\gamma \neq \gamma^{\prime}\right)$. Then it is easily checked (see Lemma) that

$$
\psi_{\gamma}=\left(\phi_{\gamma} \cdot f_{\gamma}\right) \mid F_{\gamma} \text { and } \psi_{\gamma \gamma^{\prime}}=\left(\phi_{\gamma^{\prime}} \cdot f_{\gamma \gamma^{\prime}}\right) \mid F_{\gamma}
$$

are continuous displacements of $F_{\gamma}$ in $I$. Consequently (Theorem A(iii)) $I \backslash F_{\gamma}$ contains $c$ points of $\psi_{\gamma} F_{\gamma}$ and $I \backslash F_{\gamma}$ contains $c$ points of $\psi_{\gamma \gamma^{\prime}} F_{\gamma}$, and so $S_{\gamma} \backslash F_{\gamma}$ contains $c$ points of $f_{\gamma} F_{\gamma}$ and $S_{\gamma^{\prime}} \backslash F_{\gamma^{\prime}}$ contains $c$ points of $f_{\gamma \gamma^{\prime}} F_{\gamma}$. However, this is a contradiction, since $f_{\gamma} F_{\gamma}$ and $f_{\gamma \gamma^{\prime}} F_{\gamma}$ then would have weight $c$, whereas $F_{\gamma}$ has weight $\boldsymbol{\aleph}_{0}$.

Corollary. If $B(X)$ denotes the Boolean algebra of the open-andclosed subsets of the zero-dimensional compact space $X$, then $\left\{B\left(S_{\gamma}\right)\right\}_{\gamma}$ is a family of $2^{\circ}$ Boolean algebras, each of power c, without proper automorphisms.

\section{REFERENCES}

1. J. de Groot, Groups represented by homeomorphism groups, Math. Ann. 138 (1959), 80-102.

2. B. Jónsson, A Boolean algebra without proper automorphisms, Proc. Amer. Math. Soc. 2 (1951), 766-770.

3. L. Rieger, Some remarks on automorphisms of Boolean algebras, Fund. Math. 38 (1951), 209-216.

UNiversity of AMSTERdaM AND

UNIVERSITY OF FLORIDA 